\title{
Efectos de un emisario submarino de aguas "residuales" urbanas sobre comunidades de octocorales (Octocorallia: Alcyonacea) en Cuba
}

\author{
Darlenys Hernández-Muñoz, Pedro M. Alcolado \& Miguel Hernández-González \\ Instituto de Oceanología del Ministerio de Ciencia Tecnología y Medio Ambiente de Cuba, Ave. 1ra y 186 No. 18406, \\ Ciudad Habana, Cuba; darlyhm@yahoo.com
}

Recibido 15-III-2006. C Corregido 16-III-2007. Aceptado 22-VIII-2007.

\begin{abstract}
Effects of a submarine discharge of urban waste on octocoral (Octocorallia: Alcyonacea) communities in Cuba. The composition and structure of octocoral communities on coral reefs close to a submarine outfall were studied at Reparto Flores, west of Havana City, Cuba. Octocoral community changes after the deployment of the submarine outfall in 2000 were monitored from June 2002 to September 2005, taking as baseline the data existing before its construction. The area also receives the influence of the polluted river Quibú that passes through a great part of the west side of the City. Sampling was done by means of SCUBA diving, counting and identifying colonies in situ within a $1 \mathrm{~m}^{2}$ frame that was randomly placed as many times as to warrant stabilized values of Shannon and Weaver's heterogeneity index H'. In agreement with the available hydrochemical information, changes in the diversity indexes (Shannon and Weaver's heterogeneity index H', Pielou's equitability index J', and Margalef's species richness index $\mathrm{R}_{1}$ ), the Herrera-Moreno's comparative pollution index (ICC), and density of some octocoral species at a depth of $10 \mathrm{~m}$ suggest a decrease in the influence of polluters from 1989 to 2005 . Nevertheless, these indicators were affected in 2004 by a sudden intense but brief colonization of Briareum asbestinum, a species that is not typical of polluted places. At a depth of $20 \mathrm{~m}$, a co-dominance of Plexaura kuekenthali and Eunicea clavigera (resistant and non resistant to pollution, respectively) and an increase of the comparative pollution index (ICC) was observed. The increase of P. kuekenthali, a pollution indicator, suggests a rise in the pollution effect $20 \mathrm{~m}$ in depth, because of the recent impact caused by the greater closeness of the outfall mouth $50 \mathrm{~m}$ deep. Results corroborate the hypothesis about the pollution indicator character of $P$. kuekenthali. However, this could not be explored for Eunicea flexuosa (also considered a pollution-indicator) due to an intensive illegal selective extraction for lucrative handicraft purposes, which led to a remarkable decrease in its density. B. asbestinum and E. clavigera were outlined as poorly resistant to pollution. Rev. Biol. Trop. 56 (1): 65-75. Epub 2008 March 31.
\end{abstract}

Key words: bioindicators, communities, coral reefs, Cuba, gorgonians, octocorals, pollution.

Los octocorales, han sido utilizados como bioindicadores para la evaluación de la calidad ambiental, debido a que al no poder emigrar de las áreas contaminadas por su condición de organismos sésiles, reflejan las características ambientales que han tenido lugar durante su desarrollo (Herrera-Moreno 1991). Son muy escasos los estudios sobre el empleo de octocorales como indicadores de contaminación y hasta el momento han estado relacionados a áreas de Cuba y de Australia (Herrera y Alcolado 1983, Herrera-Moreno et al. 1997,
Fabricius y Alderslade 2001). Los resultados de estos trabajos recomiendan el empleo de las comunidades de este grupo como indicadoras, por la gran consistencia observada en la dominancia de ciertas especies y del comportamiento de los índices de diversidad en relación al nivel de contaminación. Por la utilidad práctica que ello implica, se consideró que era conveniente continuar explorando esta hipótesis.

En mayo del 2000, entró en funcionamiento un emisario submarino a $50 \mathrm{~m}$ de profundidad al oeste de la Ciudad de La Habana para la 
evacuación de residuales urbanos (domésticos e industriales) de parte de esta área (Reparto Flores). Anteriormente, estos residuales eran descargados en la orilla de la costa. Esto constituyó una oportunidad para profundizar aún más en las respuestas de las comunidades de octocorales a la influencia de este tipo de residuales. Esta zona recibe además la influencia del Río Quibú, que vierte al mar desechos de otras áreas urbanas. Antes de la construcción del emisario, el Instituto de Oceanología de Cuba llevó a cabo un diagnóstico ecológico a través del análisis de las comunidades de corales, algas y octocorales, entre otras, para determinar el estado de salud del ecosistema. Estos datos estuvieron disponibles en un informe del mencionado instituto (Martínez-Iglesias et al. 1997). Sobre los octocorales, sólo se contó con datos obtenidos a $20 \mathrm{~m}$ de profundidad en el sitio donde ahora yace el emisario. Estos datos constituyeron la línea base para este trabajo y para futuros monitoreos. Por otra parte, se contó con datos de 1989 a 10 m de profundidad (Herrera-Moreno 1991).

El objetivo del presente trabajo fue conocer los cambios en la composición y estructura de las comunidades de octocorales a 10 y $20 \mathrm{~m}$ de profundidad en el área del emisario submarino, para determinar el potencial como indicadores ambientales de este grupo. Los resultados de esta investigación constituyen también una línea base para estudios y monitoreos futuros.

\section{MATERIALES Y MÉTODOS}

El área de estudio se encuentra frente a la calle 180 del Reparto Flores, oeste de Ciudad de La Habana, Cuba, en un arrecife frontal de limitado desarrollo de corales, con una cobertura estimada entre 2 y $10 \%$ (González- Ferrer, sin publ.), debido a la histórica influencia de la contaminación urbana. Se seleccionaron dos estaciones, ambas sobre el trazado del emisario submarino, ubicadas a $10 \mathrm{~m}\left(23^{\circ} 06^{\prime} 01^{\prime \prime} \mathrm{N}\right.$, $82^{\circ} 28^{\prime} 00^{\prime \prime}$ W) y $20 \mathrm{~m}\left(23^{\circ} 06^{\prime} 14^{\prime \prime} \mathrm{N}, 82^{\circ} 27^{\prime} 57^{\prime}\right.$ ' W) de profundidad. En los muestreos del 2002 las estaciones se denominaron ES10 y ES20 para $10 \mathrm{~m}$ y $20 \mathrm{~m}$ de profundidad, respectivamente. En el 2004 se nombraron ES10A y ES20A y en el 2005, ES10B y ES20B.

El primer muestreo se realizó en junio 2002, el segundo en marzo 2004 y el tercero en septiembre 2005. En todos los casos se empleó buceo autónomo, con un diseño aleatorio, utilizando una cuadrícula de $1 \mathrm{~m}^{2}$ como unidad de muestreo. Para el posicionamiento del marco se empleó una cinta métrica de $100 \mathrm{~m}$ de largo que fue extendida paralelamente a la costa a una misma profundidad, una vez a cada lado del tubo del emisario (dando un total de $200 \mathrm{~m}$ de largo). Dentro de cada tramo de $10 \mathrm{~m}$ se escogió un punto de forma aleatoria. Partiendo de estos puntos, y perpendicularmente a la cinta métrica y hacia mar afuera (al norte), se extendió imaginariamente un transecto de $10 \mathrm{~m}$ de largo mediante lo que serían diez volteos consecutivos del marco. Dentro de estos transectos hechos con volteos del marco se escogieron seis posiciones aleatorias volteando el marco las veces correspondientes a dicha posiciones a partir de la cinta métrica. De esta manera se contó con una distribución aleatoria bidimensional dentro de un rectángulo de $2000 \mathrm{~m}^{2}$. Con este patrón se muestrearon tantas unidades como fueron necesarias para alcanzar o exceder en lo posible 250 colonias, tamaño mínimo de muestra requerido para una adecuada representatividad de los índices de diversidad en octocorales, según Herrera-Moreno y Alcolado (1986a, b).

En el caso de la estación a $20 \mathrm{~m}$ de profundidad, los muestreos se realizaron en la parte superior de los camellones coralinos evitando las pendientes para conservar la profundidad. De este modo, cuando el marco de muestreo caía en la pendiente del camellón o la arena, se tomaba otro punto aleatorio alternativo predeterminado. De fallar éste, por razones prácticas se escogía la posición más cercana en el camellón siguiente, con la profundidad requerida.

Las colonias fueron contadas e identificadas hasta especie, en su mayoría in situ. Las que fueron de identidad dudosa, se les cortó una rama terminal que fue guardada en una bolsa de plástico numerada para su posterior 
identificación, siguiendo los criterios de Bayer (1961).

Se determinó en cada estación el número de especies (S) a partir del punto de estabilización de las curvas acumulativas contra número de colonias contadas. Se calculó la densidad (número de colonias $/ \mathrm{m}^{2}$ ) y por medio del programa PRIMER 5 se calculó: el índice de riqueza de especies $\mathrm{R}_{1}$ (Margalef 1951; con logaritmos neperianos), el índice de heterogeneidad H' (Shannon y Weaver 1949, con logaritmos neperianos) y el índice de equitatividad J' (Pielou 1966). Con el mismo programa, utilizando la densidad de colonias, se realizó un análisis de agrupamiento según Boesch (1977) y un análisis multidimensional no paramétrico MDS. Como índice de afinidad se empleó el coeficiente de similitud de Bray Curtis. Como método de agrupación se aplicó el algoritmo promedio de grupos (UPGMA). Las especies que más contribuyeron a la diferenciación de los grupos se determinaron a través de un análisis SIMPER, utilizando como factor el año de los muestreos.

Se calculó también con fines comparativos el índice comparativo de contaminación ICC de Herrera-Moreno (1991). Éste es la suma de los porcentajes de las especies consideradas por dicho autor como resistentes a la contaminación: Eunicea flexuosa (Lamouroux, 1821), Eunicea calyculata forma typica Bayer, 1961, E. calyculata forma coronata Bayer, 1961, Eunicea mammosa Lamouroux, 1816, Eunicea tourneforti Milne Edwards y Haime, 1857, Plexaura kuekenthali Moser, 1921 y Pseudoplexaura flagellosa Houtuyn, 1772.

Para la comparación de las densidades totales de las estaciones, se empleó un análisis de varianza bifactorial, donde los factores fueron: año y profundidad. Previamente se comprobó la normalidad y homogeneidad de varianza de los datos. La detección de diferencias entre pares de medias se realizó mediante la prueba de ámbitos múltiples de Student-NewmanKeuls (SNK). Además, se seleccionaron cuatro especies que por sus características ecológicas y porcentajes de abundancia fueron de interés para la comparación de sus densidades entre estaciones y entre años: Briareum asbestinum (Pallas, 1766), E. flexuosa, Eunicea clavigera Bayer, 1961 y P. kuekenthali. A esta última especie, luego de comprobar la normalidad y homogeneidad de varianza, se le realizó el mismo procesamiento estadístico que a los datos de densidad total. En las otras tres especies, los datos no cumplieron con los criterios de normalidad y homogeneidad de varianza y no admitieron ninguna transformación. Por ello, se les aplicó el análisis de varianza no paramétrico de Kruskal Wallis. Las pruebas estadísticas mencionadas se realizaron con un nivel de significación de 0.05 , con el programa STATISTICA 6.0.

\section{RESULTADOS}

En la estación de $10 \mathrm{~m}$ de profundidad, todos los índices de diversidad disminuyeron en el 2004 en comparación con el 2002, y aumentaron en el 2005. Contrariamente, a 20 $\mathrm{m}$ de profundidad en el 2004 aumentaron $\mathrm{R}_{1}$, H' y J', pero en el 2005 todos disminuyeron (Cuadro 1). Las especies más abundantes en todos los muestreos fueron P. kuekenthali, E. clavigera y E. flexuosa, que representan en conjunto, como promedio, el $61.8 \%$ del total de las colonias por estación (Cuadro 2).

Como se observa en el MDS, en ambas profundidades las estaciones han seguido el mismo proceso de cambio, sobre todo en la primera dimensión, entre 2002 y 2005 (Fig. 1). Además, ES10 (denominada 10/02 en el MDS) se separa completamente de las demás, lo que también se aprecia en el análisis de agrupamiento (Fig. 2). Estas representaciones también permiten ver cómo las estaciones con mayor similitud entre sí son ES10A y ES20A. Las especies que más contribuyeron a la separación de los grupos, tomando como factor el año de muestreo, fueron P. kuekenthali y E. clavigera, a las que se sumó $B$. asbestinum en el 2004.

La densidad total de octocorales no mostró diferencias significativas entre profundidades para un mismo año (ANOVA Factorial $\mathrm{p}>0.05$ ). En ambas estaciones la densidad aumentó 


\section{CUADRO 1}

Indicadores comunitarios por estación y año, incluidos los resultados de los muestreos de 1989 de Herrera-Moreno (1991) a 10 m de profundidad, y los del muestreo realizado por el Instituto de Oceanología en 1997 a $20 \mathrm{~m}$

TABLE 1

Community indicators for stations and years, including the 1989 sampling results from Herrera-Moreno (1991) at $10 \mathrm{~m}$ deep and from sampling done by the Instituto de Oceanología in 1997 at $20 \mathrm{~m}$

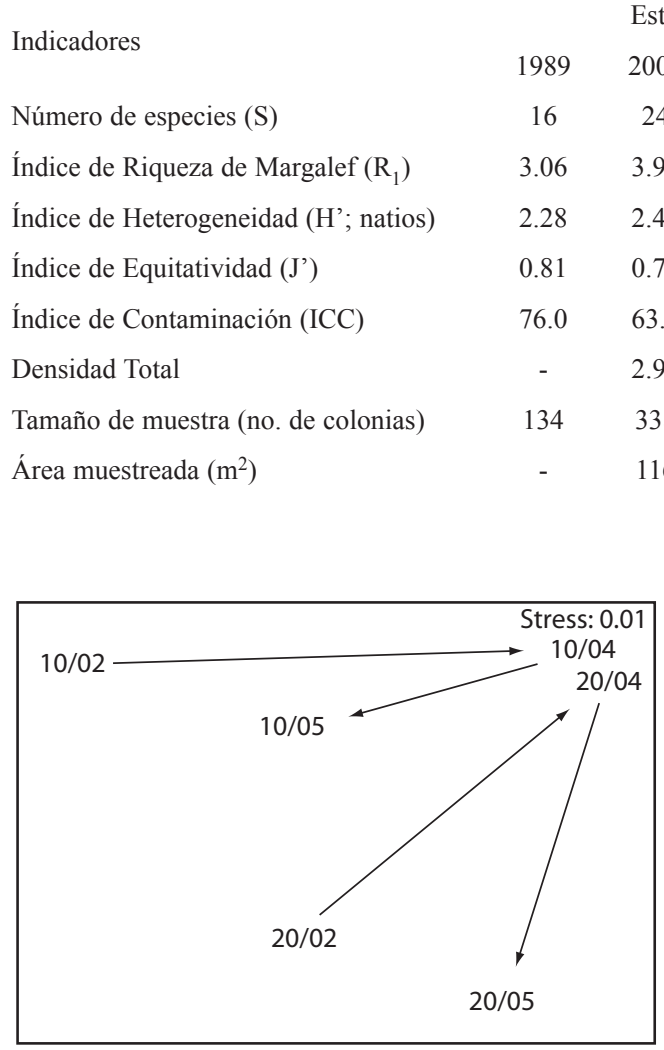

Fig. 1. MDS de las estaciones a lo largo de 2002, 2004 y 2005. Las estaciones se nombraron utilizando los números correspondientes a la profundidad y a las dos últimas cifras del año.

Fig. 1. MDS of stations along 2002, 2004 and 2005. Stations are named using the numbers corresponding to depth and to the last two figures of the year.

de forma progresiva y significativa con el paso de los años (ANOVA Factorial $\mathrm{p}<0.05$, Fig. 3). En las densidades de E. flexuosa se obtuvieron diferencias significativas entre las combinaciones de año y profundidad (Kruskal Wallis $\mathrm{p}<0.05$, Fig. 4). Lo mismo sucedió con

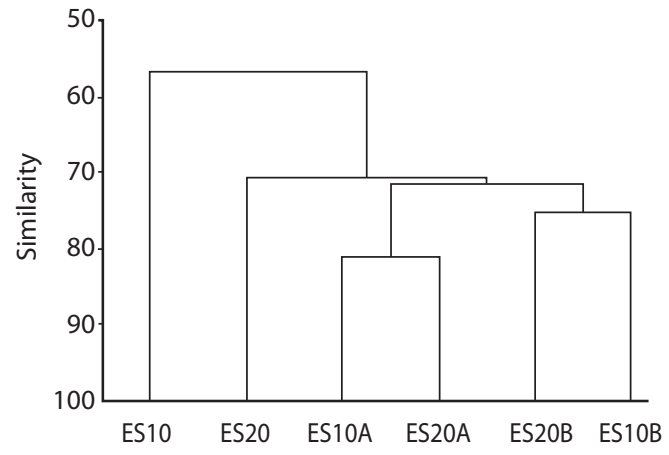

Fig. 2. Análisis de agrupamiento de las estaciones en los diferentes años.

Fig. 2. Cluster analysis of stations in the different years.

B. asbestinum (Kruskal Wallis $\mathrm{p}<0.05$, Fig. 4). En el análisis de E. flexuosa fue notorio el aumento de la densidad a $10 \mathrm{~m}$ de profundidad en 2005.

La densidad de $B$. asbestinum aumentó en 2004 en ambas profundidades. En el 2005, el 
CUADRO 2

Composición y porcentaje de abundancia en número de las especies por estación y año, incluidos los resultados de los muestreos de 1989 de Herrera-Moreno (1991) a 10 m, y los del muestreo a 20 m realizado por el Instituto de Oceanología en 1997. Las especies están ordenadas de modo decreciente acuerdo con su dominancia promedio (\%)

TABLE 2

Species composition and percent of numerical abundance for stations and years, including the 1989 sampling results from Herrera-Moreno (1991) at $10 \mathrm{~m}$ deep, and from sampling done by the Instituto de Oceanología in 1997 at $20 \mathrm{~m}$. Species are arranged in decreasing order according to their average dominance (\%)

\begin{tabular}{|c|c|c|c|c|c|c|c|c|}
\hline \multirow{2}{*}{ Especies } & \multicolumn{4}{|c|}{ Estación ES10 } & \multicolumn{4}{|c|}{ Estación ES20 } \\
\hline & 1989 & 2002 & 2004 & 2005 & 1997 & 2002 & 2004 & 2005 \\
\hline Plexaura kuekenthali & 25.37 & 28.40 & 35.80 & 29.77 & 27.50 & 43.58 & 34.59 & 52.16 \\
\hline Eunicea clavigera & 1.24 & 10.95 & 21.79 & 22.98 & 31.25 & 27.16 & 13.91 & 17.99 \\
\hline Eunicea flexuosa & 22.39 & 12.72 & 4.67 & 13.92 & 15.00 & 4.48 & 10.53 & 7.19 \\
\hline Eunicea calyculata f. typica & 2.98 & 10.06 & 9.73 & 6.47 & 2.50 & 3.28 & 14.66 & 1.44 \\
\hline Briareum asbestinum & 0.00 & 1.18 & 15.18 & 3.88 & 4.38 & 4.78 & 13.16 & 9.35 \\
\hline Eunicea calyculata f. coronata & 2.24 & 4.73 & 0.39 & 4.53 & 3.13 & 2.99 & 1.13 & 1.44 \\
\hline Pseudoplexaura flagellosa & 6.71 & 0.30 & 3.11 & 1.29 & 1.88 & 2.09 & 2.63 & 4.32 \\
\hline Pseudopterogorgia americana & 5.97 & 2.96 & 1.56 & 1.94 & 0.00 & 2.09 & 0.75 & 2.88 \\
\hline Eunicea mammosa & 8.95 & 6.21 & 0.78 & 1.29 & 0.00 & 4.48 & 0.00 & 0.00 \\
\hline Pseudopterogorgia acerosa & 0.75 & 2.37 & 0.39 & 1.94 & 0.00 & 0.90 & 0.38 & 0.36 \\
\hline Pterogorgia citrina & 0.00 & 2.07 & 0.00 & 0.97 & 0.00 & 0.00 & 0.00 & 0.00 \\
\hline Gorgonia mariae & 3.73 & 5.53 & 0.00 & 0.00 & 0.00 & 0.00 & 0.00 & 0.36 \\
\hline Muriceopsis flavida & 0.70 & 1.48 & 0.78 & 2.26 & 2.50 & 1.49 & 0.38 & 1.08 \\
\hline Gorgonia ventalina & 0.00 & 1.48 & 0.39 & 0.65 & 0.00 & 0.30 & 0.00 & 0.00 \\
\hline Muricea pinnata & 0.00 & 2.07 & 0.00 & 0.00 & 0.63 & 1.19 & 0.00 & 0.00 \\
\hline Pseudoplexaura porosa & 2.24 & 0.89 & 0.78 & 0.32 & 8.13 & 0.30 & 2.63 & 0.00 \\
\hline Eunicea tourneforti & 7.46 & 1.18 & 0.78 & 1.94 & 0.00 & 0.00 & 1.50 & 0.72 \\
\hline Gorgonia flabellum & 0.00 & 0.89 & 0.78 & 0.97 & 0.00 & 0.30 & 0.00 & 0.00 \\
\hline Plexaurella dichotoma & 0.75 & 0.89 & 0.00 & 0.97 & 0.00 & 0.00 & 0.38 & 0.00 \\
\hline Pterogorgia guadalupensis & 0.00 & 1.78 & 0.78 & 1.29 & 0.00 & 0.00 & 0.00 & 0.00 \\
\hline Muricea muricata & 0.45 & 1.18 & 0.00 & 0.00 & 0.00 & 0.00 & 0.00 & 0.00 \\
\hline Eunicea succinea & 0.00 & 0.00 & 1.56 & 0.32 & 0.63 & 0.00 & 0.75 & 0.36 \\
\hline Plexaurella nutans & 0.00 & 0.30 & 0.00 & 1.29 & 0.00 & 0.00 & 1.13 & 0.00 \\
\hline Pterogorgia anceps & 0.00 & 0.30 & 0.39 & 0.00 & 0.00 & 0.00 & 0.00 & 0.00 \\
\hline Muricea laxa & 0.00 & 0.00 & 0.00 & 0.00 & 1.25 & 0.00 & 1.13 & 0.00 \\
\hline Eunicea knightii & 0.75 & 0.00 & 0.00 & 0.00 & 0.00 & 0.00 & 0.00 & 0.00 \\
\hline Eunicea laciniata & 0.00 & 0.00 & 0.00 & 0.00 & 0.63 & 0.00 & 0.00 & 0.00 \\
\hline Muricea elongata & 0.00 & 0.00 & 0.00 & 0.65 & 0.63 & 0.60 & 0.00 & 0.36 \\
\hline Eunicea fusca & 0.00 & 0.00 & 0.39 & 0.32 & 0.00 & 0.00 & 0.00 & 0.00 \\
\hline Plexaurella grisea & 0.00 & 0.00 & 0.00 & 0.00 & 0.00 & 0.00 & 0.38 & 0.00 \\
\hline Pseudopterogorgia elisabethae & 0.00 & 0.30 & 0.00 & 0.00 & 0.00 & 0.00 & 0.00 & 0.00 \\
\hline
\end{tabular}



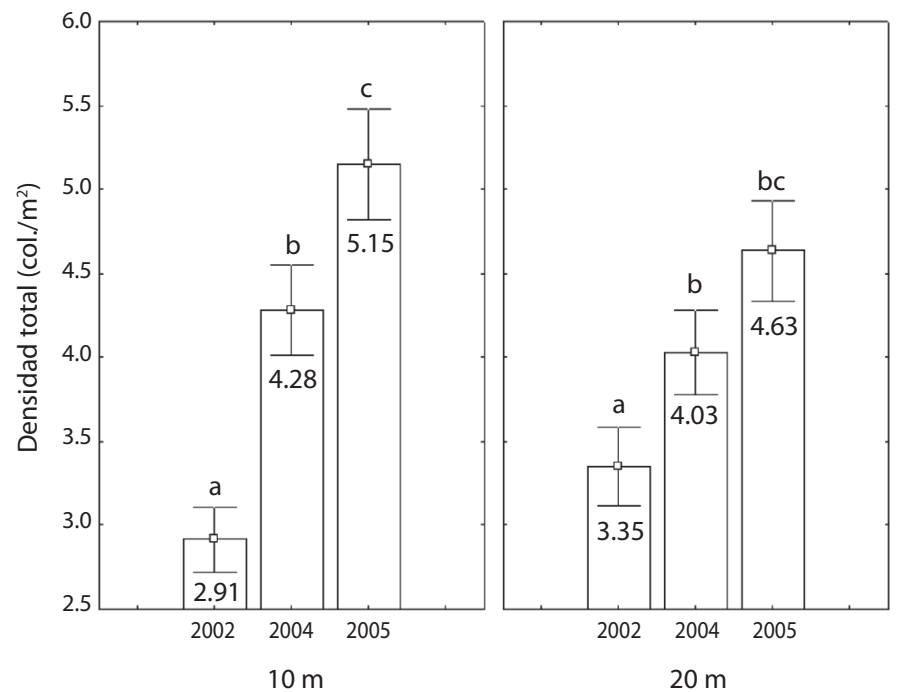

Fig. 3. Densidades totales de octocorales a 10 y $20 \mathrm{~m}$ de profundidad en 2002, 2004 y 2005.

Fig. 3. Total octocoral densities at 10 and $20 \mathrm{~m}$ deep in 2002, 2004 and 2005.
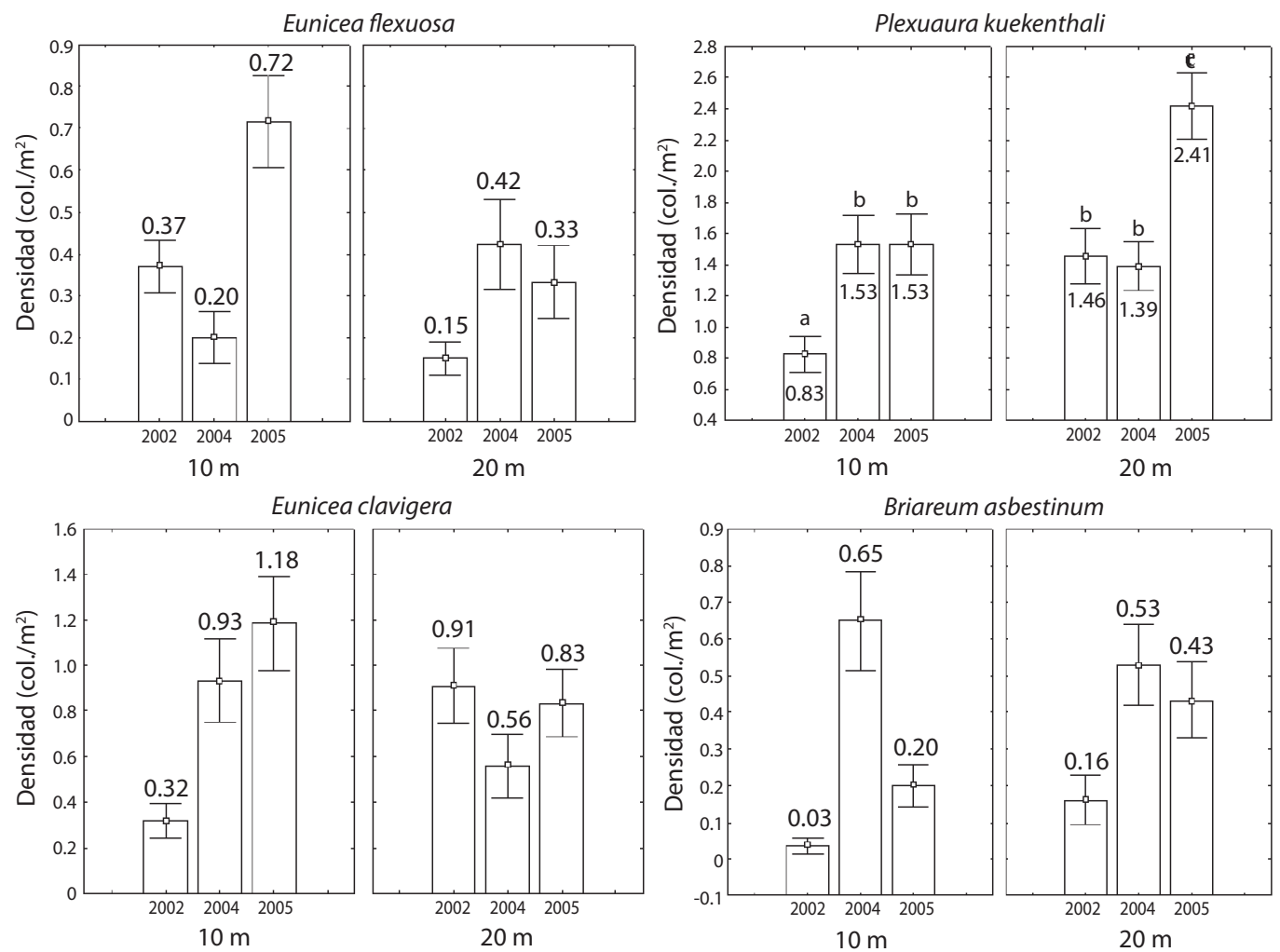

Fig. 4. Densidades de E. flexuosa, B. asbestinum, E. clavigera y P. kuekenthali a 10 y $20 \mathrm{~m}$ de profundidad en 2002,2004 y 2005.

Fig. 4. Densities of E. flexuosa, B. asbestinum, E. clavigera and P. kuekenthali at 10 and $20 \mathrm{~m}$ deep in 2002, 2004 and 2005. 
valor de la densidad fue nuevamente bajo a 10 $\mathrm{m}$. En los $20 \mathrm{~m}$ se mantuvo similar al que había alcanzado en 2004 (Fig. 4). En E. clavigera solo se encontraron diferencias significativas entre años (Kruskal Wallis, $\mathrm{p}<0.05$ ). En los 10 $\mathrm{m}$ su densidad aumentó significativamente en el 2004. A $20 \mathrm{~m}$ de profundidad, las diferencias no fueron tan notorias aunque se apreció una disminución significativa de la densidad en el 2004 (Fig. 4). Las densidades de P. kuekenthali también mostraron diferencias significativas entre años, profundidad y la combinación de ambos. Resaltó el aumento significativo de la densidad de esta especie a $20 \mathrm{~m}$ de profundidad en el 2005 (ANOVA Factorial, $\mathrm{p}<0.05$, Fig. 4).

\section{DISCUSIÓN}

El aumento de $\mathrm{S}, \mathrm{R}_{1}$ y H'a 10 m en el 2002, en comparación con los encontrados en 1989 por Herrera-Moreno (1991, Cuadro 1), sugiere una cierta mejoría en el ambiente a esta profundidad. Según Preston y Preston (1975) y Botero (1990), mayores valores de H' se corresponden generalmente con un ambiente más favorable, y según Herrera-Moreno (1991), mayores valores de $\mathrm{S}$ se relacionan generalmente con un área menos influida por la contaminación. Estos resultados, más la disminución del porcentaje total de especies resistentes a la contaminación (ICC) a esta profundidad, evidencian estar asociado a la disminución documentada de la contaminación (Montalvo et al. 2001) dada por la instalación del emisario submarino a principios del 2000.

Montalvo et al. (2001) muestran que en los siete meses muestreados antes de la puesta en funcionamiento del emisario, la concentración de fósforo inorgánico en el agua en la estación frente al Reparto Flores, varió de 0.1 a $1.5 \mu \mathrm{mol}$ (cuatro meses con más de $0.5 \mu \mathrm{mol} / 1$ ), mientras que en los cuatro meses posteriores siempre fue menor de $0.1 \mu \mathrm{mol} / \mathrm{l}$. Los cambios en el fósforo total fueron aún más importantes, con valores entre 10 y $100 \mu \mathrm{mol} / 1$. (con meses con más de $39 \mu \mathrm{mol} / \mathrm{l})$ antes de la instalación del emisario que posteriormente bajaron a concentraciones de sólo 5 a $22 \mu \mathrm{mol} / \mathrm{l}$. Las concentraciones del nitrógeno inorgánico variaron entre 1 y 5 $\mu \mathrm{mol} / \mathrm{l}$ (tres meses con más de $3 \mu \mathrm{mol} / \mathrm{l}$ ) en el primer período, mientras que en los cuatro meses muestreados después variaron aproximadamente de 1 a $3 \mu \mathrm{mol} / \mathrm{l}$ (tres meses con sólo aproximadamente $1 \mu \mathrm{mol} / \mathrm{l})$. Según datos químicos de Rodas (en prep.) estas disminuciones se mantuvieron en el 2005 (fósforo inorgánico: $0.22 \mu \mathrm{mol} / 1$, fósforo total: $0.73 \mu \mathrm{mol} / 1$, nitrógeno inorgánico: $1.57 \mu \mathrm{mol} / 1)$, lo que coincide con los autores anteriores en que la contaminación ha disminuido sustancialmente, tal como muestran las comunidades de octocorales.

Sin embargo, en un muestreo en el año 2004, Rodas et al. (2004) encontraron que, aunque los fosfatos se mantenían bajos, el nitrógeno inorgánico había aumentado respecto al 2001 (varió de 4.82 a $10.24 \mu \mathrm{mol} / \mathrm{l}$ ). Esto último pone de manifiesto que por la cercanía del contaminado río Quibú (1.25 km al este) eventualmente pueden registrarse aumentos de nutrientes de acuerdo al comportamiento de las corrientes y la magnitud de la carga contaminante de dicho río. Los indicadores de las comunidades de gorgonias, dados los tiempos de generación de éstas últimas, no se ven tan afectados por estos cambios momentáneos en la calidad del agua.

Sin embargo la disminución de $\mathrm{S}, \mathrm{R}_{1}, \mathrm{H}^{\prime}$ y J' en el 2004, no era esperada de una zona que supuestamente se encuentra en recuperación. Esto estuvo asociado a un aumento considerable del porcentaje de la especie $B$. asbestinum que, repentinamente y en un corto plazo de tiempo, pasó a ser la tercera en abundancia dentro de la comunidad, lo que denota además un crecimiento muy rápido de esta especie. Se debe tomar en cuenta que a causa del aumento en la densidad relativa de esta especie se necesitó un número menor de unidades de muestreo para alcanzar el tamaño mínimo de muestra de 250 colonias, lo que disminuyó la probabilidad de que aparecieran en las muestras otras especies que en el 2002 y en el 2005 estuvieron representadas a pesar de sus bajas densidades.

Esta colonización, al parecer fue un evento estocástico en que pudieron haber concurrido 
un éxito reproductivo, un patrón de transporte propicio y la disponibilidad de substrato, sumado a un factor determinístico como el menor nivel de contaminación existente, dado que según Herrera-Moreno (1991), B. asbestinum es una especie sensible a la contaminación y por ende pudo haber sido favorecida. El aumento de su densidad en ambas profundidades no se mantuvo en el 2005, lo que sugiere la acción selectiva de factores determinísticos locales, que tienden a llevar a las comunidades a su estado original. Se debe tener en cuenta que después de los muestreos de marzo del 2004 y antes de septiembre del 2005, pasó por Ciudad de la Habana el huracán Dennys en el verano del primer año y luego se sucedieron frentes fríos fuertes en el invierno, que pudieron haber generado un oleaje lo suficientemente fuerte como para afectar a las comunidades de octocorales principalmente a $10 \mathrm{~m}$ de profundidad y en particular a $B$. asbestinum. Según Alcolado (1981), esta especie es poco resistente a la turbulencia. En este caso, no se propone a la contaminación como otro de los factores involucrados, pues de haber sido esta uno de los elementos determinísticos, la disminución de la densidad de $B$. asbestinum hubiese sido mayor a $20 \mathrm{~m}$ porque, como será tratado más adelante, a esta profundidad parece haber actualmente un mayor efecto de contaminación que a $10 \mathrm{~m}$ y $B$. asbestinum es sensible a ésta. Los índices de diversidad a $10 \mathrm{~m}$ aumentaron nuevamente en el 2005, al desaparecer la influencia de $B$. asbestinum como especie dominante.

La inclusión de $B$. asbestinum como parte del taxoceno que se emplea como indicador ambiental parece tener inconvenientes. Hay que valorar la necesidad de exclusión de $B$. asbestinum de los estudios ecológicos de octocorales con fines bioindicadores, debido a que pertenece a un grupo taxonómico (Alcyoniina) distinto de Holaxonia y presenta un hábito morfológico diferente (lo mismo sería válido para Erithropodium caribaeorun Duchassaing y Michelotti, 1860, que no apareció en esta investigación). Al ser una especie "rastrera" y a veces incrustante, no debe reaccionar ante los tensores ambientales de la misma forma en que lo hace una colonia erecta con esqueleto córneo (Holaxonia). Además, por su forma suele ser difícil durante los conteos determinar la separación entre una colonia y otra. También es probable que, como ocurre con algunas esponjas, las colonias, ante determinada situación desfavorable, puedan fragmentarse y convertirse en varios clones independientes.

La disminución que ha sufrido el porcentaje de E. flexuosa a $10 \mathrm{~m}$ desde 1989, y que también puede estar afectando los valores de los indicadores ecológicos, debe estar dada principalmente por la extracción selectiva con fines comerciales que se ha observado en el área desde los años de crisis económica de principios de los noventa y el consecuente crecimiento del mercado privado de la artesanía. Al ser una especie resistente a la contaminación (Herrera-Moreno y Alcolado 1986a), esta disminución no parece deberse a otros tipos de cambios que podrían haberse producido en el ambiente y que desconocemos. El aumento de la densidad de E. flexuosa en el 2005 puede estar dado por un cambio en los sitios de extracción, luego de haber disminuido la densidad de la especie en el área en estudio.

Se podría pensar que la disminución de E. flexuosa a causa de su explotación también influyó en la disminución del ICC respecto a los datos de Herrera-Moreno (1991), lo que pondría en duda su efectividad como indicador de una mejoría en las condiciones en esta localidad. Sin embargo, aboga por esta mejoría el hecho de que E. clavigera, que ha ido aumentando sucesivamente, solo ha sido reportada con porcentajes significativos por Alcolado (1981) en lugares muy limpios (Sur de las provincias Granma y Santiago de Cuba, Sur de la Isla de la Juventud, Oeste del Golfo de Batabanó, entre Cabo Francés y Cabo Corrientes y en algunas áreas no contaminadas o profundas del litoral habanero donde la influencia del océano es mayor). Además, han disminuido los porcentajes de otras especies resistentes a la contaminación como E. mammosa y E. tourneforti, que no han sido objeto de extracción.

Es de interés el aumento registrado en el porcentaje y la densidad de E. clavigera a $10 \mathrm{~m}$ 
de profundidad. Este aumento puede estar relacionado en parte con la mayor dominancia de esta especie a los $20 \mathrm{~m}$ en el 2002 y que pueda haber ocurrido una dispersión de larvas de la especie desde esa profundidad hacia los 10 $\mathrm{m}$. El que su porcentaje sea cada vez mayor está sugiriendo también una disminución en la incidencia de contaminantes suficiente para que se desarrolle con éxito a esta profundidad. Sin embargo, el porcentaje y la densidad de $P$. kuekenthali, especie típica en lugares contaminados según Herrera-Moreno (1991), también ha ido aumentando aunque en menor medida en la estación a $10 \mathrm{~m}$ de profundidad, al parecer por una mejoría en la calidad del sustrato.

La comunidad de octocorales a $10 \mathrm{~m}$ de profundidad ha mostrado un proceso relativamente rápido de cambio que refleja un cierto mejoramiento en las condiciones del área para las comunidades de octocorales (aún con la colonización estocástica y temporal de $B$. asbestinum). Esto se puede inferir en el paso de una monodominancia de P. kuekenthali a una codominancia que se observa entre especies calificadas por Herrera-Moreno (1991) como resistentes (P. kuekenthali y E. calyculata forma typica) y no resistentes a la contaminación (E. clavigera y B. asbestinum). Se observó además una disminución en el curso del tiempo de los porcentajes de otras especies resistentes a la contaminación, como E. mammosa y $E$. calyculata forma typica.

En los 20 m, la disminución en el 2002 de los valores de H'y J' respecto a 1997 apareció asociada a la desaparición de E. flexuosa como especie codominante. A juzgar por la nueva disminución en los valores de los índices ecológicos a $20 \mathrm{~m}$ en el 2005 y el aumento considerable en la densidad de $P$. kuekenthali en este mismo año, parece que a esta profundidad, contrariamente a lo que debe haber ocurrido a $10 \mathrm{~m}$, se está produciendo un cierto deterioro en las condiciones ambientales, lo que debe ser verificado en muestreos futuros. Igualmente llama la atención que el porcentaje total de especies resistentes a la contaminación (ICC) en $20 \mathrm{~m}$, a diferencia de lo que observado a 10 m, haya aumentado desde 1997 hasta al 2005.
Es posible que el efecto de la presencia del desagüe del emisario, más cercano a la estación de $20 \mathrm{~m}$ que a la de $10 \mathrm{~m}$, pueda ya estarse reflejando, luego de cinco años, en un aumento de los porcentajes de P. kuekenthali y de E. calyculata forma typica, también resistente a la contaminación (Herrera-Moreno 1991). La disminución que ha tenido en el tiempo el porcentaje de E. clavigera también apunta a un deterioro en las condiciones en los $20 \mathrm{~m}$ de profundidad.

Aunque la densidad total calculada en el año 1997 no pudo ser estadísticamente comparada con las densidades totales en el 2002 y en el 2004 (por tratarse de muestreo basado en un largo recorrido pero sin replicación), dada la gran magnitud de la diferencia que se observa, se puede asumir la existencia de diferencias reales entre los distintos años.

La disminución de la densidad total en el 2002 a casi la mitad de su valor en 1997 debió estar dada en parte por eventos meteorológicos extremos, como el Huracán "Michelle", de categoría cuatro, en Noviembre del 2001. Éste atravesó Cuba con vientos máximos de 220 $\mathrm{km} / \mathrm{h}$ (Rubiera y Ballester 2002). Después de su paso se observaron numerosos octocorales arrojados a la orilla. Sin dudas, la continuada extracción selectiva de especies con fines artesanales también ha contribuido a la disminución de la densidad observada en el 2002. Aunque no se conoce la magnitud de esta acción en la zona, se podría suponer que la extracción debe haber sido elevada si se tiene en cuenta que el porcentaje de abundancia de E. flexuosa disminuyó en un tercio en el año 2002 en comparación con su valor en el año 1997 y que ésta especies es, según Alcolado (1981), una especie tolerante a la agitación. De cualquier forma no es posible discernir en qué medida influyeron ambos factores en la estructura observada en las comunidades. Las mayores densidades totales reportadas en el 2004 y en el 2005 evidencian la recuperación de las distintas poblaciones de octocorales luego del huracán. Si extrapolamos este análisis a los 10 $\mathrm{m}$, donde la afectación causada por los huracanes debe haber sido mayor, se puede explicar 
en parte el aumento progresivo que ha tenido la densidad total en los últimos años, como un proceso de recuperación luego del huracán, independientemente de las condiciones generales del ambiente.

\section{AGRADECIMIENTOS}

Agradecemos a Abel Valdivia por sus útiles aclaraciones sobre análisis multivariado, así como al Instituto de Oceanología del Ministerio de Ciencia, Tecnología y Medio Ambiente y al Centro de Investigaciones Marinas de la Universidad de La Habana por haber propiciado y apoyado esta investigación.

\section{RESUMEN}

Para profundizar en las respuestas de las comunidades de octocorales a la influencia de residuales urbanos se estudió su composición y estructura en sitios de arrecife cercanos a un emisario submarino urbano en el Reparto Flores, La Habana, Cuba. Desde junio del 2002 hasta septiembre del 2005, se efectuó el monitoreo de los cambios ocurridos en las comunidades después de la puesta en operación del emisario en el año 2000, tomando como línea base datos existentes antes del inicio de su construcción. El muestreo se realizó mediante buceo SCUBA, contando e identificando las colonias in situ dentro de cuadricula de $1 \mathrm{~m}^{2}$ de lado. Diversos índices sugieren una disminución de la influencia de la contaminación en la última década. El incremento de P. kuekenthali (indicadora de contaminación, , sugiere un aumento del efecto de la contaminación a $20 \mathrm{~m}$ de profundidad debido al impacto reciente de la mayor cercanía del desagüe del emisario que se encuentra a $50 \mathrm{~m}$ de profundidad. B. asbestinum y E. clavigera se perfilaron como poco resistentes a la contaminación.

Palabras clave: arrecifes de coral, bioindicadores, comunidades, contaminación, Cuba, gorgonias, octocorales.

\section{REFERENCIAS}

Alcolado, P.M. 1981. Zonación de los octocorales someros de Cuba y su posible uso como indicadores comparativos de tensión hidrodinámica sobre los organismos del Bentos. Inf. Cient. Téc. 187: 1-43.

Bayer, F.M. 1961. The shallow-water Octocorallia of the West Indian region. Martinicus Nijhoff, La Haya.
Boesch, D.F. 1977. Application of numerical classification in ecological investigations of water pollution. Virginia Institute of Marine Science, Special Scientific Report 77: 113.

Botero, L. 1990. Quantitative structure of Gorgonian communities in Tayrona National Park, Caribbean coast of Colombia. An. Inst. Cien. Mar Limnol. UNAM 7: 73-88.

Fabricius, K. \& P. Alderslade. 2001. Soft corals and sea fans. A comprehensive guide to the tropical shallow water genera of the central-west Pacific, the Indian ocean and the Red sea. Australian Institute of Marine Science, Townsville, Queensland, Australia.

Herrera-Moreno, A. 1991. Efectos de la contaminación sobre la estructura ecológica de los arrecifes coralinos en el litoral Habanero. Tesis de doctorado, Instituto de Oceanología, La Habana, Cuba.

Herrera-Moreno, A. \& P.M. Alcolado. 1983. Efectos de la contaminación sobre las comunidades de octocorales al Oeste de la Bahía de La Habana. Cien. Biol. 10: 69-85.

Herrera-Moreno, A. \& P.M. Alcolado. 1986a. Estructura ecológica de las comunidades de octocorales en el litoral de Mariel y su comparación con el litoral habanero. Cien. Biol. 15: 55-75.

Herrera-Moreno, A. \& P.M. Alcolado. 1986b. Estructura ecológica de las comunidades de octocorales del arrecife de Santa Cruz del Norte. Rep. Invest. Inst. Oceanol., Academia de Ciencias de Cuba 49: 1-27.

Herrera-Moreno, A., P.M. Alcolado \& P. García-Parrado. 1997. Estructura ecológica de las comunidades de octocorales en el arrecife de barrera del Rincón de Guanabo. Avicennia 6/7: 73-84.

Martínez-Iglesias, J.C., D. Ibarzábal, B. Martínez-Daranas, L. Rodas, G. Menéndez, P. García-Parrado, G. GarcíaMokina, A. Areces, R. García-santos \& A. Niévares. 1997. Informe preliminar del estudio de factibilidad de la construcción del Emisario Submarino para la evacuación de los residuales del Reparto Flores. Resultados de la primera etapa. Instituto de Oceanología, Ministerio de Ciencia, Tecnología y Medio Ambiente, La Habana, Cuba.

Margalef, R. 1951. Diversidad de especies en las comunidades naturales. Publ. Inst. Biol. Aplic. Barcelona 9: $5-27$.

Montalvo, J.F., M. Cano, J. Díaz, R. Pérez, S.C. Esponda, M.E. Cesar, C.M. Peón y J. Rendón. 2001. Nutrientes en la zona litoral entre los ríos Quibú y Jaimanitas, Ciudad de 
la Habana, Cuba. VII Taller Contribución a la educación ambiental y la protección ambiental, Cátedra de Medio Ambiente. Instituto Superior de Ciencias y Tecnologías Nucleares, Ministerio de Ciencia, Tecnología y Medio Ambiente de Cuba 2: 12-17.

Pielou, E.C. 1966. The measurement of diversity in different types of biological collections. J. theoretical Biol. 13: 131-144.

Preston, E.M. \& J.L. Preston. 1975. Ecological structure in a West Indian gorgonian fauna. Bull. Mar. Sci. 25: 248-258.

Rodas, L., S. Cerdeira, A. Areces, J. Montalvo, S. Losa, R. Valle, Y. Delgado, M. Soza, L. Rivas, R. Guerra,
S. González, Z. Marcos, J. Gutiérrez, S. Lorenzo, I. García, M. Sánchez, R. Núñez, M.E. César, S. Esponda, V. Delgado, R. García, J.L. Hernández, M. Hernández \& A. Niévares. 2004. Informe Final Calidad Ambiental de la Zona Costera al Oeste de Ciudad de la Habana. Instituto de Oceanología, La Habana, Cuba.

Rubiera, J.M. \& M. Ballester. 2002. Temporada ciclónica 2001 en el Atlántico Norte. Departamento de Pronósticos. INSMET- CITMA. Inst. Meteor., La Habana, Cuba.

Shannon, C.E. \& W. Weaver. 1949. The Mathematical Theory of Communication. University of Illinois, Urbana, EEUU. 
